\title{
Minimum JT Duration
}

National Cancer Institute

\section{Source}

National Cancer Institute. Minimum IT Duration. NCI Thesaurus. Code C62116.

The minimum duration (time) of the JT interval, obtained from a set of measurements of the JT interval. The JT interval is defined as the time from the J point (end of ventricular depolarization, the point at which the QRS meets the ST segment) to the end of the T wave (representing the end of ventricular repolarization). 